\title{
Communications
}

\section{ESMO today}

\section{Aktuelle Neuigkeiten vom ESMO-Kongress}

Bereits zum dritten Mal berichtet der Online-Informationsservice ESMO today vom Kongress der European Society for Medical Oncology (ESMO), der dieses Jahr in München stattfindet. Fachärzte haben damit die Möglichkeit, die vorgestellten aktuellen Forschungsergebnisse zeitnah online abzurufen. Ab dem 20. Oktober, 22 Uhr, fassen sechs deutsche Onkologen auf www.esmo-today.de Ergebnisse verschiedener Fachgebiete zusammen. Die Experten wählen interessante Studien zu ihrem jeweiligen Themenschwerpunkt aus, besuchen vor Ort die Vorträge und bereiten die Daten in kompakten und informativen Videobeiträgen auf.

\section{Themen und Referenten von ESMO today} 2018:

Gastrointestinale Tumoren - Prof. Dr. med. Dirk Arnold, Medizinischer Vorstand des Asklepios Tumorzentrums Hamburg und Direktor der Klinik für Onkologie mit Sektion Hämatologie und Palliativmedizin, AK Altona

Lungenkarzinome - Prof. Dr. med. Frank Griesinger, Direktor der Klinik für Hämatologie und Onkologie, Universitätsklinik Innere Medizin-Onkologie, Pius-Hospital, Medizinischer Campus Universität Oldenburg

Kopf- und Halstumoren - Prof. Dr. med. Viktor Grünwald, Professor für Interdisziplinäre Uroonkologie, Universitätsklinikum Essen

Fortgeschrittenes Mammakarzinom - Prof. Dr. med. Christian Jackisch, Chefarzt der Klinik für Gynäkologie und Geburtshilfe, Sana-Klinikum Offenbach

Dermatologische Tumoren - Prof. Dr. med. Dirk Schadendorf, Direktor der Klinik für Dermatologie und Direktor des Westdeutschen Tumorzentrums (WTZ), Universitätsklinikum Essen

Urologische Tumoren - Prof. Dr. med. Arnulf Stenzl, Ärztlicher Direktor der Klinik für Urologie, Universitätsklinikum Tübingen

Für interessierte Nutzer besteht zudem die Möglichkeit, sich für den Newsfeed zu registrieren, um per E-Mail über tagesaktuelle Themen von ESMO today informiert zu werden. Die Anmeldung erfolgt über die Internetseite www.esmo-today.de oder per E-Mail an info[at] esmo-today.de

\section{Examens de spécialiste}

Examen de spécialiste en vue de l'obtention du titre de spécialiste en gastroentérologie

Partie A: Examen écrit: European Board of Gastroenterology and Hepatology Knowledge Examination

Date et lieu: 10 avril 2019 à différents endroits en Suisse (Genève, Zurich, ev. Lausanne)

Partie B: Examen pratique oral avec des discussions de cas

Date et lieu: 30 avril 2019, Hôpital Uster, Division de Gastroentérologie

\section{Délai d'inscription:}

Partie A: 19 décembre 2018 jusqu'au 16 janvier 2019 par http://www.ebghe.eu

Partie B: jusqu'au 31 janvier 2019

Vous trouverez de plus amples informations sur le site web de l'ISFM www.siwf.ch

$\rightarrow$ Domaines spécialisés $\rightarrow$ Titres de spécialiste et formations approfondies (formation postgraduée) $\rightarrow$ gastroentérologie
Examen en vue de l'obtention de la formation approfondie en hépatologie à adjoindre au titre de spécialiste en gastroentérologie

Date et lieu:

Partie A/B/C: 30 avril 2019. Examens écrits et examen pratique oral à l'hôpital Uster, Division de Gastroentérologie

\section{Inscription jusqu'au 31 janvier 2019}

L'inscription est définitive dès le paiement de la taxe d'examen

Vous trouverez de plus amples informations sur le site web de l'ISFM www.siwf.ch $\rightarrow$ Domaines spécialisés $\rightarrow$ Titres de spécialiste et formations approfondies (formation postgraduée) $\rightarrow$ Gastroentérologie 